\title{
Isolation prevalence of pulmonary nontuberculous mycobacteria in Ontario in 2007
}

\author{
Mohammed Al Houqani MBBS FRCPC 1 , Frances Jamieson MD FRCPC ${ }^{2,3}$, Pamela Chedore MLT ${ }^{4}$, \\ Mauli Mehta ${ }^{1}$, Kevin May MLT ${ }^{4}$, Theodore K Marras MD FRCPC 5
}

\begin{abstract}
M Al Houqani, F Jamieson, P Chedore, M Mehta, K May, TK Marras. Isolation prevalence of pulmonary nontuberculous mycobacteria in Ontario in 2007. Can Respir J 2011;18(1):19-24.
\end{abstract}

BACKGROUND: The reported prevalence of pulmonary nontuberculous mycobacteria (NTM) infections is increasing.

OBJECTIVE: To determine the 'isolation prevalence' of NTM in 2007 and compare it with previously published research that examined the increasing rates of isolation of NTM from clinical pulmonary specimens between 1997 and 2003.

METHODS: Isolation prevalence was investigated retrospectively by reviewing a cohort of all positive pulmonary NTM culture results from the Tuberculosis and Mycobacteriology Laboratory, Public Health Laboratory (Toronto, Ontario) in 2007, which identifies at least 95\% of NTM isolates in Ontario. Isolation prevalence was calculated as the number of persons with a pulmonary isolate in a calendar year divided by the contemporary population and expressed per 100,000 population. Changes in isolation prevalence from previous years were assessed for statistical significance using generalized linear models with a negative binomial distribution.

RESULTS: In 2007, 4160 pulmonary isolates of NTM were collected from 2463 patients. The isolation prevalence of all species (excluding Mycobacterium gordonae) was 19 per 100,000 population in 2007 - an increase from previous observations reported for Ontario - corresponding to an average annual increase of $8.5 \%$ from 1997 to 2007 ( $\mathrm{P}<0.0001)$. Average annual increases in isolation prevalence of Mycobacterium avium complex (8.8\%, $\mathrm{P}<0.0001)$ and Mycobacterium xenopi $(7.3 \%, \mathrm{P}=0.0005)$ were largely responsible for the overall increase, while prevalence rates of rapidly growing mycobacteria remained relatively stable.

CONCLUSION: The isolation prevalence of pulmonary NTM continues to increase significantly in Ontario, supporting the belief that pulmonary NTM disease is increasingly common.

Key Words: Atypical; Epidemiology; MAC; Mycobacterium infections; NTM; Ontario

\section{La prévalence d'isolement des mycobactéries pulmonaires non tuberculeuses en Ontario en 2007}

HISTORIQUE : La prévalence déclarée d'infections pulmonaires mycobactériennes non tuberculeuses (MBN) est à la hausse.

OBJECTIF : Déterminer la «prévalence d'isolement » des infections MBN en 2007 et la comparer avec des recherches déjà publiées qui portaient sur les taux croissants d'isolement des infections MBN dans des échantillons pulmonaires cliniques entre 1997 et 2003.

MÉTHODOLOGIE : Les chercheurs ont examiné rétrospectivement la prévalence d'isolement en examinant une cohorte de résultats de cultures pulmonaires MBN positives provenant du Tuberculosis and Mycobacteriology Laboratory, Public Health Laboratory de Toronto, en Ontario, en 2007, qui dépiste au moins $95 \%$ des isolats pulmonaires MBN en Ontario. Les chercheurs ont calculé la prévalence d'isolement comme le nombre de personnes ayant un isolat pulmonaire au cours d'une année civile, divisé par la population contemporaine et exprimé sur 100000 habitants. Ils ont évalué les modifications à la prévalence d'isolement par rapport aux années précédentes pour en établir la signification statistique au moyen des modèles linéaires généralisés selon une distribution binomiale négative.

RÉSULTATS : En 2007, les chercheurs ont colligé 4160 isolats pulmonaires d'infections MBN chez 2463 patients. La prévalence d'isolement toutes espèces confondues (à l'exception du Mycobacterium gordonae) était de 19 cas pour 100000 habitants en 2007, une augmentation par rapport aux observations antérieures déclarées en Ontario, correspondant à une augmentation annuelle moyenne de 8,5 \% entre 1997 et $2007(\mathrm{P}<0,0001)$. Les augmentations annuelles moyennes de prévalence d'isolement du complexe Mycobacterium avium $(8,8 \%, \mathrm{P}<0,0001)$ et Mycobacterium xenopi $(7,3 \%, P=0,0005)$ étaient largement responsables de l'augmentation globale, tandis que les taux de prévalence de mycobactéries à croissance rapide demeuraient relativement stables.

CONCLUSION : La prévalence d'isolement d'infections pulmonaires MBN continue d'augmenter considérablement en Ontario, ce qui étaye la conviction que la maladie pulmonaire $\mathrm{MBN}$ est de plus en plus courante.

Epidemiological studies of pulmonary NTM infection are challenging for several reasons. First, NTM are commonly isolated from environmental sources; therefore, NTM growth in culture may be due to specimen contamination (2). Second, pulmonary NTM isolation does not necessarily imply disease because positive cultures may only represent colonization, or an early or indolent infection of uncertain significance (2). Finally, NTM is not a reportable disease in many jurisdictions; consequently, studies of NTM epidemiology often comprise small or incomplete samples. As identified in a previous systematic review (3), many epidemiological studies of pulmonary NTM showed that its prevalence varies according to geographical region and is increasing significantly. The 'isolation prevalence' of pulmonary NTM in Ontario was found to have increased from 9.1 per 100,000 population in 1997 , to 14.1 per 100,000 populations in 2003 - corresponding to an average annual increase of $8.4 \%$ (4). A slight decrease in the isolation prevalence of pulmonary NTM was observed in Ontario in 2003

fulfill criteria for NTM pulmonary disease (2).

${ }^{1}$ University of Toronto, Toronto; ${ }^{2}$ Clinical and Environmental Microbiology; ${ }^{3}$ Ontario Agency for Health Protection and Promotion;

${ }^{4}$ Tuberculosis and Mycobacteriology Laboratory, Public Health Laboratory - Toronto; ${ }^{5}$ Joint Division of Respirology, University Health

Network and Mount Sinai Hospital, Department of Medicine, University of Toronto, Toronto, Ontario

Correspondence: Dr Theodore K Marras, Toronto Western Hospital, 7E-452, 399 Bathurst Street, Toronto, Ontario M5T 2 S8.

Telephone 416-603-5767, fax 416-603-5375, e-mail ted.marras@utoronto.ca 
(from 16.5 per 100,000 population to 14.1 per 100,000 population), raising questions about the subsequent direction of pulmonary NTM isolation prevalence (4). Furthermore, the increases in pulmonary NTM prevalence in Ontario and elsewhere have not been adequately explained. It was postulated that the increase is due, in part, to increased awareness, which has led to more investigations and/or improved laboratory methods.

In the present follow-up retrospective cohort review study, our objectives were twofold. First, we sought to address whether the previously observed increase in pulmonary NTM isolation prevalence has continued. Second, to investigate whether heightened clinical suspicion has driven the observed increase in isolation prevalence, we assessed whether there was a recent change in the annual number of specimens submitted for mycobacterial culture, and the annual fraction of mycobacterial cultures that were positive for NTM.

\section{METHODS}

To study the isolation prevalence of pulmonary NTM in Ontario, data were collected retrospectively from the Public Health Laboratory (PHL) in Toronto, Ontario. The number of patients with pulmonary specimens yielding a positive NTM culture in 2007 were determined. The PHL-Toronto Tuberculosis and Mycobacteriology laboratory processes the majority of specimens for mycobacterial culture in Ontario, and also acts as a reference laboratory for species identification of cultures from most other laboratories in the province. Laboratory directors and senior technologists from other Ontario laboratories that identify NTM organisms were contacted to estimate the fraction of provincial NTM isolates that are identified in the Toronto Ministry of Health TB laboratory. From 1999 to 2003, other laboratories reported approximately 200 isolates of NTM per year. Based on these results, during this time period, more than $90 \%$ of isolates were estimated to be identified at the PHL-Toronto TB Laboratory. Due to changes in practices at other laboratories, this fraction increased very slightly in the final year that the present study examined, when the PHL-Toronto TB Laboratory likely identified approximately $95 \%$ of Ontario NTM isolates. Therefore, it was decided that excluding isolates from other laboratories would not significantly alter frequency estimates and, therefore, only data from the PHL-Toronto TB laboratory were included in the present study.

All specimens submitted for mycobacterial analysis in Ontario are routinely processed using acid-fast bacilli smear and microbiological culture methods. Specimen culture was performed using the Bactec 460 TB system (Becton Dickinson Microbiology Systems, USA) until May 2000 and, thereafter, with the Bactec MGIT 960 system (Becton Dickinson Microbiology Systems). Specimens were processed and analyzed as described by Hanna et al (5). NTM cultures were speciated using DNA probes (AccuProbe, Gen-Probe, USA) for Mycobacterium avium complex (MAC) and Mycobacterium gordonae, and by highperformance liquid chromatography for other species (6).

The isolation prevalence of pulmonary NTM was calculated as the total number of patients with an NTM isolate divided by the contemporary provincial population. To study whether the previous increase in isolation prevalence had continued, isolation prevalence was compared with previous data (4). The isolation prevalence was compared among years by visually inspecting graphical data. Statistical significance was assessed with generalized linear models with a negative binomial distribution using prevalence as the outcome variable and year as the predictor variable. This was performed using SAS Proc Genmod (SAS Institute Inc, USA) with a negative binomial distribution and a logarithmic link function. Regression analysis was also performed using SAS Proc Autoreg (SAS Institute Inc) to verify the lack of autocorrelation. The above calculations were repeated for the four most commonly isolated species or species groups in each year except for $M$ gordonae. Although it is a common isolate, $M$ gordonae was excluded because it may be a contaminant from water ingested before collecting sputum, and is infrequently associated with clinically significant disease (7). NTM prevalence was compared with the contemporary TB case rates in Ontario.
Based on the number of specimens that were submitted for mycobacterial culture between 1997 and 2007, the possibility of bias regarding increases in the isolation prevalence was assessed. Because there were no changes in the laboratory techniques used to detect mycobacteria in Ontario between 2003 and 2007, it was reasoned that a general increase in awareness of pulmonary NTM would have led to an increase in the number of mycobacterial culture requests, but not necessarily an increase in the measured isolation prevalence.

Finally, the small decrease in isolation prevalence that was observed in 2003 was considered to have been possibly related to the outbreak of severe acute respiratory syndrome (SARS) that occurred in Toronto during the second quarter of 2003 (8). It was hypothesized that the drastic reduction in physician visits throughout the Toronto area during the SARS outbreak led to fewer assessments for possible NTM. A reduction in the number of specimens submitted would be a useful surrogate measure to support this hypothesis. Therefore, the monthly and quarterly number of specimens submitted to the PHL TB laboratory for mycobacterial culture during the year before and after the outbreak were studied to assess for the possible SARS-related reduction in early 2003.

Data were entered directly into an electronic database (Access 2000, Microsoft Corproration, USA). All statistical analyses were performed using $\mathrm{SAS}^{\mathrm{R}}$ version 8.02 (SAS Institute Inc) and Epi Info 2000 (Centers for Disease Control and Prevention, USA). Ethics approval was granted by the appropriate institutional review board.

\section{RESULTS}

During 2007, 4364 NTM isolates including all body sites were collected from 2602 patients. Limiting specimens to pulmonary sites yielded 4160 isolates from 2463 patients. Ninety-five per cent of all NTM isolates were from a pulmonary source and $95 \%$ of all patients with NTM isolates had specimens from a pulmonary source. Table 1 summarizes the number of isolates and individual patients according to anatomical site from 1997 to 2003 (4).

Of the $5 \%$ of patients who harboured NTM isolated from nonpulmonary sites, $12 \%$ (17 of 139 ) were from blood, $15 \%$ (21 of 139 ) were from urine and $73 \%$ (101 of 139) were from specimens from unspecified anatomical sites. The number of patients with pulmonary isolates in 2007 was greater than in previous years. MAC was isolated in $62 \%$ of cases, Mycobacterium xenopi was isolated in 23\%, rapidly growing mycobacteria (Mycobacterium abscessus, Mycobacterium chelonae and Mycobacterium fortuitum) were isolated in $8 \%$ and Mycobacterium kansasii was isolated in $2 \%$.

Isolation prevalence and temporal trends of pulmonary NTM, and the contemporary provincial rates of TB are presented in Table 2 . The isolation prevalence for the four most common NTM species combined was 19 per 100,000 population - more than double the isolation prevalence in 1997, which represented a statistically significant increase $(\mathrm{P}<0.0001)$ and corresponded to an average annual increase of $8.5 \%$. Similar increases in prevalence were observed for each individual NTM species (Table 2) and were all statistically significant. Isolation prevalence for 2007 and the previous years of study (1997 to 2003) are presented in Figure 1. Isolation prevalence of pulmonary NTM continued to increase while the incidence of TB in Ontario continued to slowly decrease (Figure 1). The isolation prevalence of pulmonary NTM was greater than the rate of TB throughout the study period, and the ratio of NTM isolation prevalence to TB case prevalence increased during the study period.

To investigate whether the increase in isolation prevalence was due to a surveillance bias, the annual change in the number of respiratory specimens submitted for mycobacterial culture between 1997 and 2007, and differences in the fraction that were positive for NTM were examined. Table 3 summarizes the number of pulmonary specimens submitted to the PHL-Toronto TB laboratory between 1997 and 2007, as well as the number and fraction that were positive for NTM. The annual number of respiratory specimens submitted for mycobacterial culture generally increased throughout the study period, with the 
TABLE 1

Number of nontuberculous mycobacteria isolates and patients with isolates per year according to anatomical site

\begin{tabular}{|c|c|c|c|c|c|c|}
\hline \multirow[b]{2}{*}{ Year } & \multicolumn{6}{|c|}{ Isolates, $\mathbf{n} /$ patients, $\mathbf{n}$ (anatomical source of specimen) } \\
\hline & Pulmonary & Blood & Urine & Bone marrow & Unknown & Total $^{*}$ \\
\hline 1998 & $1890 / 1214$ & $32 / 23$ & $4 / 4$ & $1 / 1$ & $285 / 249$ & $2212 / 1433$ \\
\hline 2000 & $2519 / 1641$ & $29 / 20$ & $10 / 8$ & $0 / 0$ & $131 / 114$ & $2689 / 1755$ \\
\hline 2001 & $3218 / 2043$ & $25 / 20$ & $1 / 1$ & $0 / 0$ & $105 / 92$ & $3349 / 2126$ \\
\hline 2002 & $3471 / 2319$ & $23 / 14$ & $5 / 4$ & $0 / 0$ & $137 / 114$ & $3636 / 2410$ \\
\hline 2007 & $4160 / 2463$ & $36 / 17$ & $23 / 21$ & $0 / 0$ & $145 / 101$ & $4364 / 2602$ \\
\hline
\end{tabular}

*Total number of patients does not equal the sum of columns or rows due to patients with isolates from different anatomical sites, the presence of multiple species or repeatedly positive cultures over the years

TABLE 2

Annual isolation prevalence of pulmonary nontuberculous mycobacteria and reported tuberculosis cases in Ontario from 1997 to 2003 , and 2007

\begin{tabular}{|c|c|c|c|c|c|c|c|}
\hline \multirow[b]{3}{*}{ Year } & \multirow{3}{*}{$\begin{array}{c}\text { Ontario } \\
\text { population*, } \\
\mathbf{n} \times 1000 \\
\end{array}$} & \multicolumn{5}{|c|}{ Prevalence $^{\dagger}, \%$ (total cases, $n$ ) } & \multirow[b]{3}{*}{ Tuberculosis" } \\
\hline & & \multicolumn{3}{|c|}{ Mycobacterium } & \multirow{2}{*}{$\begin{array}{l}\text { Rapidly growing } \\
\text { mycobacteria }^{\ddagger}\end{array}$} & \multirow[b]{2}{*}{ All species ${ }^{\S}$} & \\
\hline & & Avium complex & Xenopi & Kansasii & & & \\
\hline 1997 & $11,168.4$ & $5.6(629)$ & $2.6(293)$ & $0.09(10)$ & $1.2(134)$ & $9.1(1020)$ & $6.9(776)$ \\
\hline 1999 & $11,506.4$ & $7.2(824)$ & $3.4(388)$ & $0.17(20)$ & $1.4(165)$ & $11.8(1360)$ & $6.0(696)$ \\
\hline 2000 & $11,685.4$ & $7.6(892)$ & $3.3(389)$ & $0.22(26)$ & $1.7(194)$ & $12.5(1465)$ & $6.0(696)$ \\
\hline 2001 & $11,897.6$ & $9.4(1115)$ & $4.0(474)$ & $0.32(38)$ & $2.0(240)$ & $15.0(1780)$ & $5.8(688)$ \\
\hline 2003 & $12,238.3$ & $8.4(1027)$ & $4.2(513)$ & $0.23(28)$ & $2.0(240)$ & $14.1(1734)$ & $5.4(657)$ \\
\hline 2007 & $12,803.9$ & $11.9(1521)$ & $4.4(565)$ & $0.31(40)$ & $1.6(201)$ & $19.2(2463)$ & $5.1(654)$ \\
\hline Average annual change, $\%$ & N/A & 8.8 & 7.3 & 17.4 & 3.8 & 8.5 & -4.3 \\
\hline$P^{\star \star *}$ & N/A & $<0.0001$ & 0.0005 & 0.006 & 0.12 & $<0.0001$ & $<0.0001$ \\
\hline
\end{tabular}

*Values for 1999 to 2003, and 2007 from Statistics Canada (http://www.statcan.ca), values for 1997 and 1998 were estimated by back extrapolation; ${ }^{\dagger}$ Prevalence for nontuberculous mycobacteria species defined as the isolation prevalence and the prevalence of tuberculosis was defined according to reported cases of tuberculosis; ${ }^{\ddagger}$ Mycobacterium (M) abscessus, chelonae and fortuitum; ${ }^{\S}$ Data for $\mathrm{M}$ avium complex, $\mathrm{M}$ xenopi, $\mathrm{M}$ fortuitum, $\mathrm{M}$ chelonae, $\mathrm{M}$ abscessus and $\mathrm{M}$ kansasii combined; "Total number of tuberculosis cases from Public Health and Epidemiology Reports Ontario $(15,16)$. Rate calculated according to population from Statistics Canada; ${ }^{\star *} P$ value for comparing prevalence over time using generalized linear models with a negative binomial distribution. N/A Not applicable

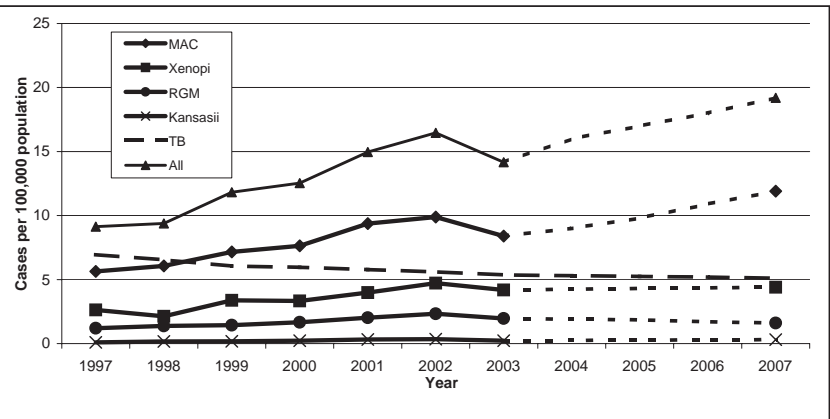

Figure 1) Prevalence of pulmonary nontuberculous mycobacteria (NTM) infections and tuberculosis (TB) case rates in Ontario from 1997 to 2003, and 2007. Dashed lines represent interpolated values. The isolation prevalence rates of all NTM infections significantly increased, while TB rates significantly decreased ( $P \leq 0.0001$ for all). $T B$ rates were calculated from the total number of $T B$ cases (references 10 to 12) and population data from the Statistics Canada website for 1999 to 2007 (http://www.statcan.cal) and estimated by back extrapolation (for 1997 and 1998). The comparison of NTM and TB rates is limited: NTM data represent isolation prevalence, while TB data represent the incidence of all new cases (including culture-negative cases). Kansasii Mycobacterium kansasii; MAC Mycobacterium avium complex; RGM Rapidly growing mycobacteria; Xenopi Mycobacterium xenopi
TABLE 3

Number of pulmonary specimens submitted for mycobacterial analysis in Ontario per year (1997 to 2007)

\begin{tabular}{|c|c|c|c|c|}
\hline \multirow[b]{2}{*}{ Year } & \multicolumn{2}{|c|}{ Pulmonary specimens } & \multicolumn{2}{|c|}{ Pulmonary isolates } \\
\hline & Submitted, n & $\begin{array}{c}\text { Annual } \\
\text { change, \% }\end{array}$ & $\mathbf{n}$ & $\begin{array}{c}\text { Positive for } \\
\text { NTM, \% }\end{array}$ \\
\hline 1997 & 15,405 & - & 1658 & 10.8 \\
\hline 1998 & 19,632 & 23.0 & 1890 & 9.6 \\
\hline 1999 & 23,898 & 21.7 & 2407 & 10.1 \\
\hline 2000 & 30,749 & 28.6 & 2519 & 8.2 \\
\hline 2001 & 35,612 & 15.8 & 3218 & 9.0 \\
\hline 2002 & 40,885 & 14.8 & 3471 & 8.5 \\
\hline 2003 & 37,482 & -8.3 & 3200 & 8.5 \\
\hline 2004 & 40,736 & 8.6 & NA & NA \\
\hline 2005 & 38,740 & -4.8 & NA & NA \\
\hline 2006 & 39,798 & 2.7 & NA & NA \\
\hline 2007 & 38,600 & -3.0 & 4160 & 10.8 \\
\hline
\end{tabular}

NA Not available; NTM Nontuberculous mycobacteria

exception of a decrease in the number of specimens submitted in 2003, compared with the previous year. Thereafter, the annual number of specimens submitted remained relatively constant. The annual fraction of specimens that yielded NTM was relatively stable, ranging from $8.2 \%$ to $10.8 \%$, with no clear temporal trend between 1997 and 2007. An analysis of the annual fraction of mycobacterial cultures that yielded NTM failed to detect an obvious indiscriminant surveillance 


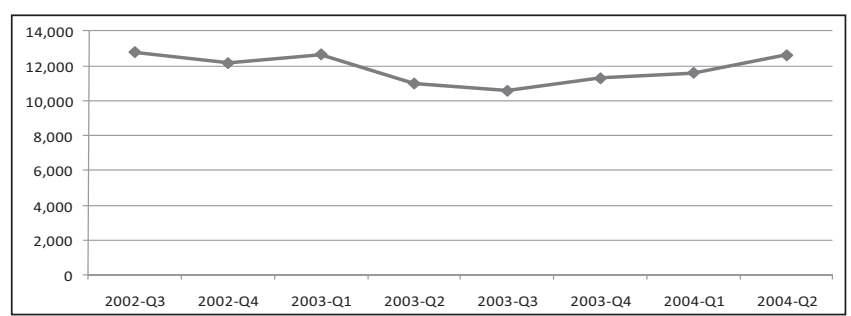

Figure 2) Total number of specimens submitted to the Toronto Public Health Laboratory (Toronto, Ontario) for mycobacterial culture according to calendar quarter $(\mathrm{Q})$. The outbreak of severe acute respiratory syndrome occurred in Toronto in the second Q of 2003 (2003-Q2)

bias. An indiscriminant surveillance bias would be expected to be associated with a very large increase in the number of specimens submitted for culture and a reduced fraction of positive specimens. The frequency of microscopy smear-positive NTM cases from 1997 to 2003 was previously assessed (4), with a similar analysis extended to 2007. The frequency of smear-positive, NTM culture-positive specimens ranged from a low of $10.5 \%$ in 1997 , to a high of $17.4 \%$ in 2000 , and was $12.1 \%$ in 2007 . There was no obvious temporal trend in the fraction of smear-positive cases. Although there is no perfect correlation between smear status and NTM disease and, if smear-positive status is a reasonable surrogate for NTM disease, then the relatively stable fraction of cases with smear-positive isolates over time suggests that the frequency of NTM disease probably increased during the study period.

Considering the isolation prevalence over the years, 2003 appeared to be slightly anomalous because it was the only year studied that yielded a smaller number of patients with isolates than the previous year (Table 2; Figure 1). To assess whether the small decrease in isolation prevalence in 2003 may have been related to the SARS outbreak that occurred in Toronto in the same year, the number of specimens submitted to the PHL-Toronto TB laboratory for mycobacterial culture according to month and quarter during the year before and the year after the SARS outbreak was studied. Because the SARS outbreak was associated with a drastic reduction in physician visits throughout the Toronto area, it was reasoned that there were likely fewer assessments for possible NTM during this time period. A reduction in the number of specimens submitted would, therefore, be a useful surrogate measure to support this idea. The average number of specimens per quarter that were submitted to the PHL-Toronto TB Laboratory during the four quarters before the start of the SARS outbreak in Toronto (April to June 2002, to January to March 2003) was 12,679. During the quarter of the SARS outbreak (April to June 2003), 10,999 specimens were submitted (a reduction of 13\%). During consecutive quarters from the start of the SARS outbreak, specimen submission (reduction from previous average) was 10,582 (17\% reduction), 11,296 ( $11 \%$ reduction) and 11,610 ( $8 \%$ reduction). Subsequently, there was a return to a similar rate of specimen submission. This is presented graphically in Figure 2. Compared with 2002, there was a reduction of $8.3 \%$ in the number of specimens submitted for mycobacterial culture in 2003, and a $13 \%$ reduction in the number of NTM isolates.

\section{DISCUSSION}

The isolation prevalence of pulmonary NTM in Ontario continues to increase. Based on previous studies $(3,4,9,10)$, the median rates of pulmonary NTM isolation are approximately 6.2 per 100,000 population in North America, 8.3 per 100,000 population in Europe, 15 per 100,000 population in Asia, 7.2 per 100,000 population in Australia and 6.7 per 100,000 population in British Columbia. Our results represent a remarkably higher prevalence than generally reported in North American studies and studies from most other areas. We observed a significantly higher isolation prevalence in 2007 (19.2 per 100,000 population) compared with 2003 , the final year of our previously reported study (4) (14.1 per 100,000 population). Although the comparison of NTM with TB is limited because NTM data reflect isolation prevalence and TB data reflect the incidence of new cases (including culture-negative cases), it is interesting to note that the gap between the rates of TB isolation and pulmonary NTM isolation continues to widen, with a further reduction in the rates of TB from 2003 to 2007 (from 5.4 per 100,000 population in 2003 to 5.1 per 100,000 population in 2007) (11-13).

The high isolation prevalence and ongoing increase over time is an important observation that is consistent with results of previous studies. All well-defined, population-based studies that presented data on the frequency of pulmonary NTM isolation or disease over time have reported increases (14-18). Published data are consistent with our results and suggest that pulmonary NTM infection is a growing problem.

Increases in the frequency of pulmonary NTM colonization and disease throughout the world have not been well explained; however, several possible explanations have been proposed (18-21). It was suggested that the increase may have been due to a higher awareness of pulmonary NTM disease among clinicians that led to more referral and specimen submission for NTM culture. Our data argue against an 'indiscriminant surveillance bias', wherein a much larger number of specimens might be referred for culture and, despite a lower fraction of positive cultures, a larger absolute number of positive cultures may have resulted. Over the study period, we observed an increase in the total number of specimens submitted for culture, but no change in the fraction that yielded NTM. If the fraction of culture-positive specimens from peripheral laboratories decreased, then our estimate of overall culture-positive frequency over time may be incorrect. Approximately 200 isolates were processed annually in peripheral laboratories between 1999 and 2003. If the culture-positive fraction in peripheral laboratories decreased by $50 \%$ (from $10 \%$ to $5 \%$ ), then the calculated effect in total fraction of positive specimens in Ontario would likely be less than $2 \%$, which is insufficient to indicate growing surveillance bias. An 'informed surveillance bias', however, could explain some of our results. This may have occurred if clinicians were using another sensitive test more frequently to identify patients who likely harboured pulmonary NTM, and subsequently obtained specimens from such patients only. An example of such a test is chest computed tomography (CT) scanning. There may have been an increase in the use of chest CT scans during the study period, and physicians would have reacted appropriately to an observation of characteristic features of pulmonary NTM with the collection of specimens for mycobacterial studies. Admittedly, it is very difficult to postulate how the total number of specimens requested may relate to clinicians' suspicion for NTM because we suspect that the indication for the majority of the requests is to rule out TB. It has also been suggested that improved laboratory methods have led to an apparent increase in the frequency of pulmonary NTM. However, our findings argue strongly against the possibility that this hypothesis explains the increase during the study period. The PHL-Toronto TB Laboratory has used the same mycobacterial growth system (Bactec MGIT 960) since 2000, and the frequency of NTM isolation has continued to increase. In addition, rates of TB in Ontario are decreasing despite the fact that the same microbiological culture systems are also used to detect Mycobacterium tuberculosis complex (11).

We found that the small reduction in isolation prevalence between 2002 and 2003 was mirrored closely by the reduction in specimens submitted for mycobacterial studies. The timing of the reduction in specimen submission was closely tied to the SARS outbreak and the associated reduction in physician visits in many ambulatory and inpatient settings (22). It appears that the small reduction in pulmonary NTM isolation prevalence between 2002 and 2003 was due to the collection of fewer specimens rather than a reduction in the number of infected people in the population.

The isolation prevalence of pulmonary NTM in Ontario was driven mainly by the large increase in MAC isolation. An explanation for this finding is not apparent from our results; however, future 
research will hopefully be able to address this interesting observation. MAC was also recently reported to be the most common NTM isolated in British Columbia (10). Unlike other parts of North America, $M$ xenopi was the second most common NTM isolated in Ontario, which comprised $20 \%$ to $25 \%$ of prevalent cases in our study. The ongoing high prevalence of $M$ xenopi compared with other parts of North America $(14,16,23-27)$ underscores the importance of the geographical heterogeneity of pulmonary NTM infection. Other studies have shown a substantial proportion of $\mathrm{M}$ xenopi among cases of pulmonary NTM infection in southern Ontario $(28,29)$ and northwestern New York, USA (30). It is possible that the common source of water (ie, Lake Ontario - a large freshwater lake) among most of the cases reported here, and previously in Southern Ontario and northwestern New York, is relevant.

Unfortunately, our study could not identify causes of the increase in the isolation prevalence of pulmonary NTM. The changes in the frequency of pulmonary NTM isolation could be due to host changes, pathogen changes or an interaction between host and pathogen. Host changes leading to increased susceptibility could be an important factor. There may be more immunocompromised patients due to HIV infection, malignancy or advanced age. However, there are relatively few data in this regard. Pathogen changes (ie, mutations toward more pathogenic strains) could play an important role, but we are unaware of any data demonstrating changes in 'ambient' environmental NTM pathogenicity over time. Finally, it is possible that people are being increasingly inoculated with NTM through exposure to water or soil aerosols. Although difficult to study, a change from predominantly tub-bathing to predominantly shower-bathing over the past decades has possibly driven some of the increase.

Our study is limited by the lack of clinical information. Therefore, we can only comment on the isolation prevalence of pulmonary NTM, rather than the clinical significance of isolates in a given patient. The number of people with 'disease' versus 'colonization' cannot be determined from the present study. However, based on a previous review (3), if one assumes that approximately $25 \%$ of people with a pulmonary NTM isolate have 'disease', then the corresponding disease prevalence in Ontario would be approximately five per 100,000 population, ie, approximately the same as the annual incidence rate of TB. Given that the disease duration of TB is likely relatively short compared with that of pulmonary NTM disease, we very strongly suspect that the prevalence of pulmonary NTM disease is significantly greater than the prevalence of TB in Ontario. We also limited our assessment to the isolation prevalence of pulmonary NTM, as in our previous report. Because of the presumed chronic nature of pulmonary NTM disease, it may be challenging to identify a new or incident case without an adequate 'look-back' period to ensure that a patient was not previously harbouring a pulmonary NTM isolate. In the present study, we did not analyze data from 2004 to 2006; therefore, we could not determine how many of the patients identified in 2007 may have already had the organism identified in one of the previous three years. However, review of the data from 1997 to 2003 identified only a very small minority of people with isolates in more than one year. Because pulmonary NTM disease is a chronic disease lasting for years, we suspect that a large proportion of patients do not have regular follow-up specimens collected every year. For this reason, the observed isolation prevalence may be an underestimate. If there was a steady increase in the use of routine follow-up cultures, the observed increase in isolation prevalence could represent a biased impression of trends therein. The data for such an analysis are not available, but the review of data from 1997 to 2003 did not suggest increasingly frequent surveillance. In the present study, we also lacked data from some Ontario laboratories that identify NTM species. We included only isolates from the PHLToronto TB laboratory, which processes more than $95 \%$ of respiratory specimens submitted for mycobacterial culture in Ontario. Isolates in other laboratories were not included unless they were submitted to PHL-Toronto; therefore, the isolation prevalence that we measured is likely an underestimate. Approximately $3 \%$ of specimens were not identified according to the anatomical site of origin. We suspect that the source of some of these specimens was pulmonary and, therefore, our results may represent a further small underestimate of the isolation prevalence of pulmonary NTM. Overall, we believe that the limitations of our population-based study suggest that our measurements underestimate the isolation prevalence of pulmonary NTM infection, and that the true prevalence is higher than we measured.

Our population-based study revealed that the isolation prevalence of pulmonary NTM in Ontario continues to increase and that it is higher than previously reported. The increased prevalence is largely driven by the increase in the isolation of MAC. The high and consistently increasing prevalence we observed suggests that the burden of pulmonary NTM infection is significant and will continue to rise. However, we cannot rule out an informed surveillance bias that could explain some of the increase that was observed. Further work is needed to understand the frequency of disease among all patients harbouring pulmonary NTM, and to continue to measure the frequency of NTM isolation over time to understand whether the current strong upward trends continue.

\section{REFERENCES}

1. Huard RC, Lazzarini LC, Butler WR, et al. PCR based method to differentiate the subspecies of the Mycobacterium tuberculosis complex on the basis of genomic deletions. J Clin Microbiol 2003;41:1637-50.

2. Griffith DE, Aksamit T, Brown-Elliott B, et al. An official ATS/IDSA statement: Diagnosis, treatment and prevention of nontuberculous mycobacteria disease. Am J Respir Care Med 2007;175:367-416.

3. Marras TK, Daley CL. Epidemiology of human pulmonary infection with nontuberculous mycobacteria. Clin Chest Med 2002;23:553-67.

4. Marras TK, Chedore P, Ying AM, Jamieson F. Isolation prevalence of pulmonary 1997-2003 non-tuberculous mycobacteria in Ontario. Thorax 2007;62:661-6.

5. Hanna BA, Ebrahimzadeh A, Elliott LB, et al. Multicenter evaluation of the BACTEC MGIT 960 system for recovery of mycobacteria. J Clin Microbiol 1999;37:748-52.

6. Butler WR, Guthertz LS. Mycolic acid analysis by high-performance liquid chromatography for identification of Mycobacterium species. Clin Microbiol Rev 2001;14:704-26.

7. Arnow PM, Bakir M, Thompson K, Bova JL. Endemic contamination of clinical specimens by Mycobacterium gordonae. Clin Infect Dis 2000;31:472-6.

8. World Health Organization. Cumulative Number of Reported Probable Cases of Severe Acute Respiratory Syndrome (SARS). $<$ http://www.who.int/csr/sars/country/en/index.html> (Accessed on January 30, 2009).

9. Haverkort F. National atypical mycobacteria survey, 2000. Commun Dis Intell 2003;27:180-9.

10. Hernández-Garduño E, Rodrigues M, Elwood RK. The incidence of pulmonary non-tuberculous mycobacteria in British Columbia, Canada. Int J Tuberc Lung Dis 2009;13:1086-93.

11. Vik J. Epidemiology of tuberculosis in Ontario, 1999-2001. Public Health and Epidemiology Report, Ontario 2003;14:105-15.

12. Ministry of Health and Long-Term Care. Summary of reportable diseases in Ontario - 4th quarter 2003. Public Health and Epidemiology Report Ontario, 2004;15:73-5.

13. Tuberculosis in Canada 2007 - Pre-release. <http://www. publichealth.gc.ca/tuberculosis> (Accessed on January 7, 2011).

14. Robakiewicz M, Grzybowski S. Epidemiological aspects of non-tuberculous mycobacterial disease and of tuberculosis in British Columbia. Am Rev Respir Dis 1974;109:613-20.

15. du Moulin GC, Sherman IH, Hoaglin DC, Stottmeier KD. Mycobacterium avium complex, an emerging pathogen in Massachusetts. J Clin Microbiol 1985;22:9-12.

16. Isaac-Renton JL, Allen EA, Chao CW, Grzybowski S, Whittaker EI, Black WA. Isolation and geographic distribution of Mycobacterium other than M tuberculosis in British Columbia, 1972-81. CMAJ 1985;133:573-6.

17. Debrunner M, Salfinger M, Brandli O, von Graevenitz A. Epidemiology and clinical significance of nontuberculous mycobacteria in patients negative for human immunodeficiency virus in Switzerland. Clin Infect Dis 1992;15:330-45. 
18. Kubin M, Svandova E, Medek B, Chobot S, Olsovsky Z. Mycobacterium kansasii infection in an endemic area of Czechoslovakia. Tubercle 1980;61:207-12.

19. Sakatani M. [Nontuberculous mycobacteriosis; the present status of epidemiology and clinical studies.] Kekkaku 1999;74:377-84.

20. Donnabella V, Salazar-Schicchi J, Bonk S, Hanna B, Rom WN. Increasing incidence of Mycobacterium xenopi at Bellevue Hospital: An emerging pathogen or a product of improved laboratory methods? Chest 2000;118:1365-70.

21. Prince DS, Peterson DD, Steiner RM, et al. Infection with Mycobacterium avium complex in patients without predisposing conditions. N Engl J Med 1989;321:863-8.

22. Stukel TA, Schull MJ, Guttmann A, et al. Health impact of hospital restrictions on seriously ill hospitalized patients: Lessons from the Toronto SARS outbreak. Med Care 2008;46:991-7.

23. Griffith DE, Girard WM, Wallace RJ Jr. Clinical features of pulmonary disease caused by rapidly growing mycobacteria: An analysis of 154 patients. Am Rev Respir Dis 1993;147:1271-8.
24. Ahn CH, Lowell JR, Onstad GD, Shuford EH, Hurst GA. A demographic study of disease due to Mycobacterium kansasii or M intracellulare-avium in Texas. Chest 1979;75:120-5.

25. O'Brien RJ, Geiter LJ, Snider DE Jr. The epidemiology of nontuberculous mycobacterial diseases in the United States. Results from a national survey. Am Rev Respir Dis 1987;135:1007-14.

26. Good RC. Isolation of non-tuberculous mycobacteria in the United States, 1979. J Infect Dis 1980;142:779-83.

27. Good RC, Snider DE Jr. Isolation of nontuberculous mycobacteria in the United States, 1980. J Infect Dis 1982;146:829-33.

28. Contreras MA, Cheung OT, Sanders DE, Goldstein RS. Pulmonary infection with nontuberculous mycobacteria. Am Rev Respir Dis 1988;137:149-52.

29. Simor AE, Salit IE, Vellend H. The role of Mycobacterium xenopi in human disease. Am Rev Respir Dis 1984;129:435-8.

30. Jiva TM, Jacoby HM, Weymouth LA, Kaminski DA, Portmore AC. Mycobacterium xenopi: Innocent bystander or emerging pathogen? Clin Infect Dis 1997;24:226-32. 


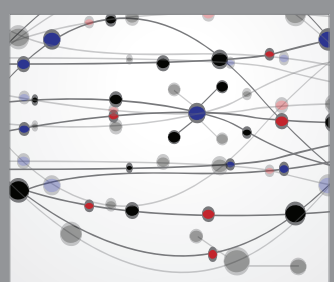

The Scientific World Journal
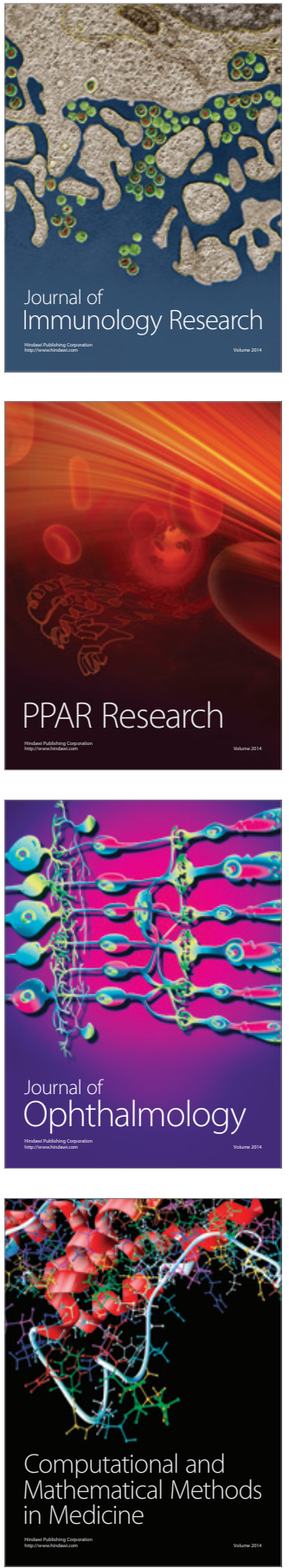

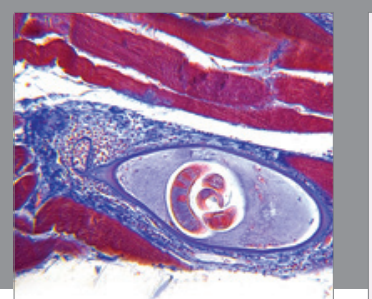

Gastroenterology Research and Practice

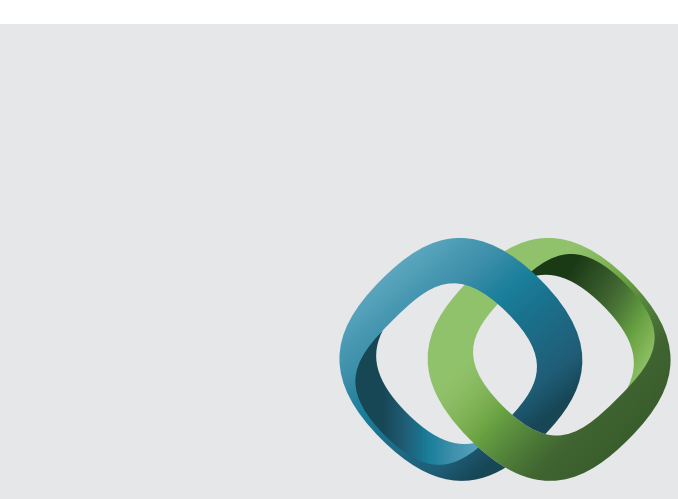

\section{Hindawi}

Submit your manuscripts at

http://www.hindawi.com
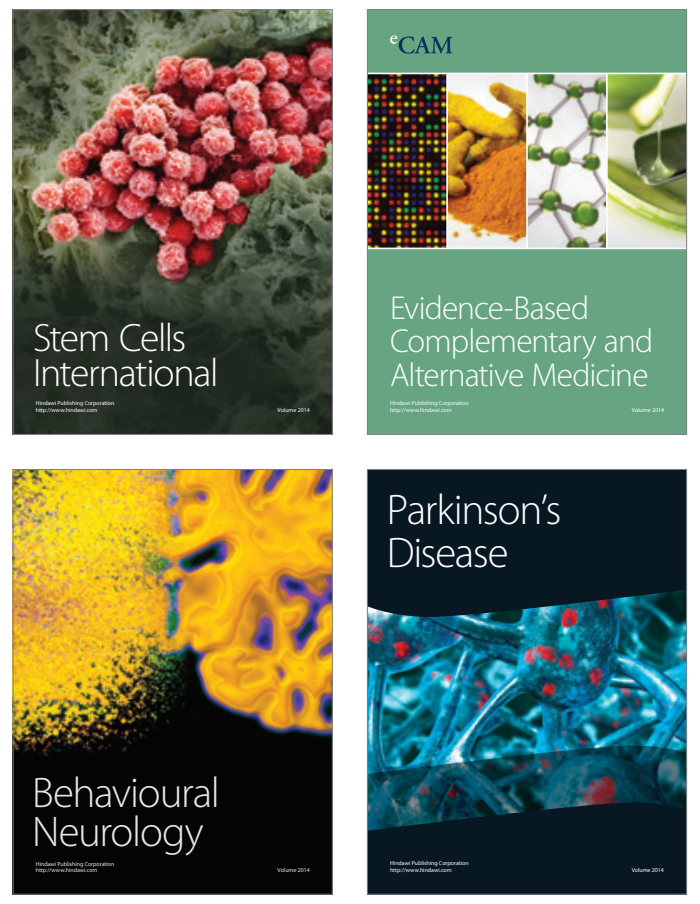
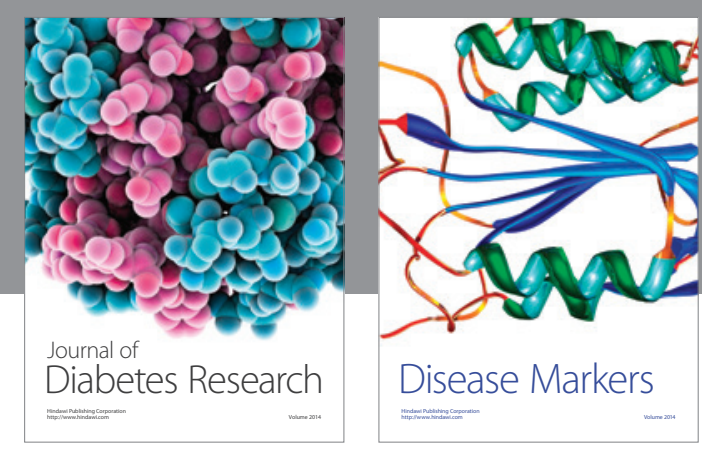

Disease Markers
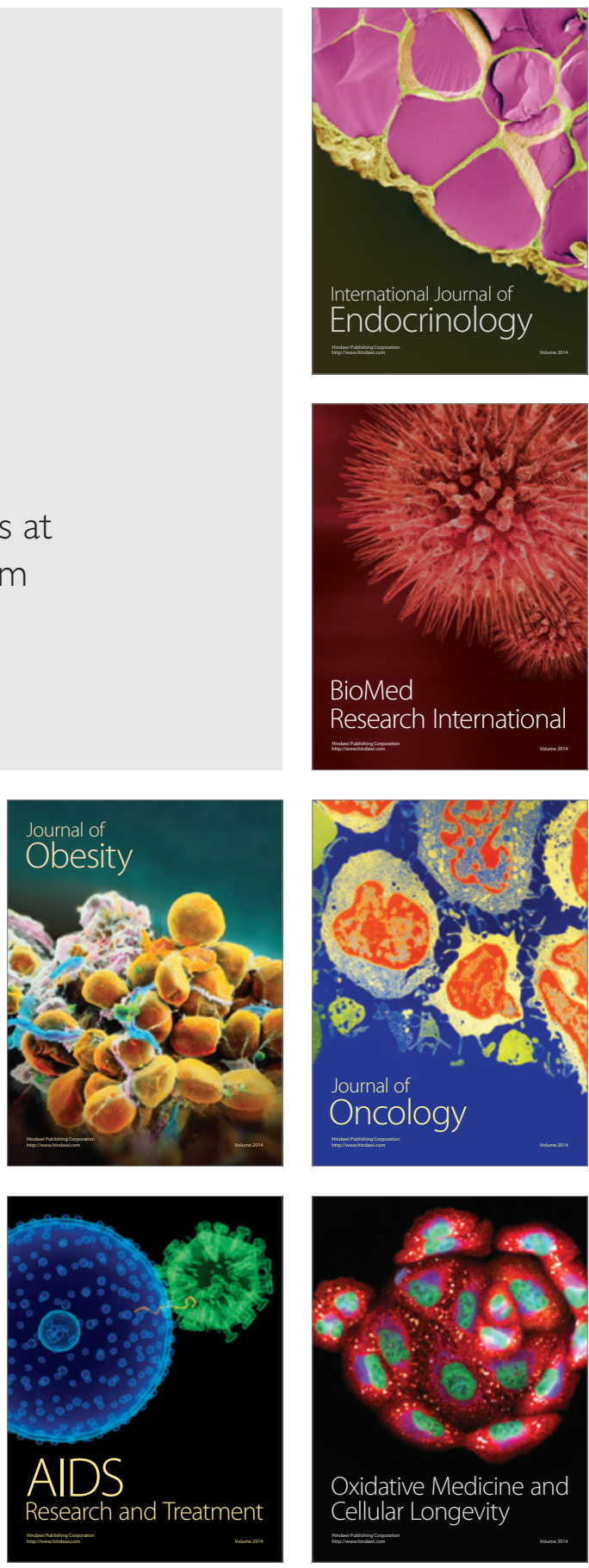